\title{
Effect of Tendon and Bulk Vibration of The Soleus Muscle on the Threshold and Positive Slope of the H-Reflex Recruitment Curve
}

\author{
Amin KORDI YOOSEFINEJAD ${ }^{1}$, Giti TORKAMAN², Seyed Mohammad FIROOZABADI ${ }^{3}$ \\ ${ }^{1}$ School of Rehabilitation, Shiraz University of Medical Sciences, Shiraz, Iran \\ 2Department Physical Therapy, Faculty of Medical Sciences, Tarbiat Modares University, Tehran, Iran \\ ${ }^{3}$ Department Medical Physics, Faculty of Medical Sciences, Tarbiat Modares University, Tehran, Iran
}

\begin{abstract}
Objective:Muscle tone abnormalities are one of the main problems of patients with central nervous system disease. There are several methods to normalize muscle tone. One of them is vibration therapy. Applying vibration to different parts of a muscle may display different responses, but the differences have not yet been studied. The aim of this study was to evaluate these potential differences.

Material and Methods: Participants were twelve healthy men. All participants took part in four experiments, twice as control and twice as experiment group. In the experiment group, vibrator (frequency: $100 \mathrm{~Hz}$, amplitude: $1.6 \mathrm{~mm}$ ) was applied to the bulk of soleus for $1 \mathrm{~min}$ in one session and to the tendon in another session. In the control group, the inoperative vibrator was put on the same landmarks on the bulk and tendon of the muscle so as to have the same pressure and tactile stimuli.

Results: Statistical analysis showed that vibration applied to the bulk and tendon of soleus enhanced the positive slope and threshold of the $\mathrm{H}$-reflex recruitment curve.

Conclusion: Applying vibration with these parameters increased the recruitment of fast and slow motor neurons. Apart from enhanced motor neuron recruitment, the inhibitory action of Renshaw cells declined because of supraspinal and segmental effects.

Keywords: Vibration, H-reflex, recruitment curve, positive slope, threshold
\end{abstract}

\section{Introduction}

One of the main problems of patients with central nervous system (CNS) disorders is muscle tone imbalance. Tone imbalance can manifest as spasticity and hypotonia.

Upper motor neuron (MN) lesions are associated with muscle tone abnormalities (1). There are various physical therapy techniques to normalize muscle tone. One of these techniques is the application of vibration that can have facilitatory or inhibitory effects (2). Vibration frequencies of more than $200 \mathrm{~Hz}$ can damage the skin. Frequencies more than $150 \mathrm{~Hz}$ cause pain and discomforts but frequencies less than $75 \mathrm{~Hz}$ have inhibitory effect on muscles (1). Vibration can be applied to both agonists and antagonists of a given muscle group. To facilitate a hypotonic muscle, the muscle belly is first stretched and the vibratory stimuli can be used to further stretch the muscle. To inhibit a hypertonic muscle, the antagonist muscle can be vibrated (2).

Applying mechanical vibration to a muscle tendon in relaxed human subjects induces either a contraction of the vibrated 
muscle or contraction of the antagonist muscle group $(3,4)$. Hreflex is used to study the effects of vibration on MN pool excitability (5-7). H-reflex is a monosynaptic reflex that can show the changes of $\mathrm{MN}$ recruitment. Continuous vibration applied over limb muscles causes depression of the tendon jerks and $\mathrm{H}$-reflexes of those muscles $(8,9)$. Morin et al. $(10)$ showed that the application of a 10-ms burst of vibration to the tendon of tibialis anterior depressed the facilitation of the soleus H-reflex produced by the homonymous group I volley, with the subthreshold for the $\mathrm{H}$-reflex delivered $3 \mathrm{~ms}$ earlier.

The most common parameter of the $\mathrm{H}$-reflex that is studied is the $\mathrm{H}$-reflex amplitude $(11,12)$, but there are many controversies associated with it (13-19). The H-reflex recruitment curve is a better parameter that provides a more precise evaluation of MN excitability $(20,21)$. The study of the slope and threshold of the H-reflex recruitment curve may provide some information about the MN excitability.

Lyshenko et al. (22) studied recurrent inhibition of forearm flexor MNs by evaluating peak-to-peak H-reflex amplitude. They showed that vibration disinhibits the recurrent inhibition of Renshaw cells. This can be attributed to segmental and suprasegmental effects on MNs or Renshaw cells.

Beekhuizen and her coworkers studied the effects of acute whole-body vibration (WBV) on the soleus H-reflex. They showed that changes of spinal la neuron excitability occur acutely after WBV. Vibration is known to have an effect on presynaptic la neuron inhibition, making this a likely mechanism for the observed change in the H-reflex amplitude (23). Armstrong et al. (24) studied the acute effect of WBV on H-reflex of 19 college-aged subjects. They concluded that factors such as training specificity and muscle fiber type might contribute to the differing $\mathrm{H}$-reflex responses, and the effect of WBV on specific performance measures should be interpreted with the understanding that there may be considerable variation among individuals.

No previous study has compared the effects of the direct application of bulk and tendon vibration on the $\mathrm{H}$-reflex recruitment curve; therefore, this study investigated the effects of applying mechanical vibration $(100 \mathrm{~Hz})$ to the soleus muscle belly and Achilles tendon on $\mathrm{MN}$ excitability.

\section{Material and Methods}

Twelve healthy non-athlete men volunteers without a history of neuromuscular disorders (mean age $29 \pm 4$ years) participated in this study. The study was approved by the ethical commission of Tarbiat Modares University in accordance with the Helsinki declaration of 1975, revised 2002. Informed consent was obtained from all the participants. All participants were asked to relax their muscles as much as possible during the study period, and the same position was maintained throughout the experiments. Participants were asked to have sufficient rest the night before the experiments because fatigue has a detrimental effect on the amplitude of the H-reflex. In order to have reliable data, 10 maximum $\mathrm{H}$-reflexes were elicited and recorded for each subject. If $\mathrm{H}$-reflex peak-to-peak amplitude changes were more than $10 \%$, the participant was excluded from the analysis. The room temperature was kept between $23^{\circ} \mathrm{C}$ and $24^{\circ} \mathrm{C}$. To elimi- nate probable circadian effects, all experiments were performed between 8 and 12 a.m.

The vibrator used (WAHL 4196, USA), which has two different frequencies of 20 and $100 \mathrm{~Hz}$. To verify the operating parameters of the vibrator, we calibrated it with a Signal Enhancement Seismograph (LCM-4) at the Geography Laboratory of Tarbiat Modares University. At the Frequency of $100 \mathrm{~Hz}$, the amplitude was $1.6 \mathrm{~mm}$.

For data collection, we used a computer-controlled stimulator with an isolator (Nihon Kohden ss-104j, Japan), an amplifier with a frequency window of 3-10 KHz to record the H-reflex, an IBM-compatible computer with an analog-to-digital (A/D) board (12-bit, 33-KHz sampling rate). Computer software written on $\mathrm{C}^{++}$was used to record the data (square wave: $700-\mu$ s pulse width and $0.2-\mathrm{Hz}$ frequency).

Each participant took part in this study on 4 separate days, separated by 2 -day intervals, twice in the control group and twice in the experimental group. After measurement of the skin temperature, the participant was asked to lie down in a prone position. The active recording electrode was placed at the midpoint between the popliteal fossa and the medial malleolus, and the inactive electrode was placed two centimeters distal to the active electrode. The stimulating bar electrode was placed on the popliteal fossa slightly towards the medial aspect (cathode in proximal and anode in distal) and the ground electrode was placed between the recording and the stimulating electrodes.

First, the stimulation intensity was gradually increased to record the maximum H-reflex $\left(\mathrm{H}_{\text {max }}\right)$. Then, by increasing the intensity, the maximum $\mathrm{M}$-wave $\left(\mathrm{M}_{\max }\right)$ was recorded when $\mathrm{H}$-reflex was minimal. Finally, we decreased the intensity to find the minimum $\mathrm{H}$ response (threshold of the H-reflex recruitment curve). Three points between the first and second intensities and three others between the second and third intensities were selected for in order to record the H-reflex. On the whole, the recruitment curve consisted of 45 reflexes elicited at different nine electrical stimulation intensities (5 successive recorded $\mathrm{H}$-reflexes in each intensity). A sample of $\mathrm{H}$-reflex recruitment curve is shown in Figure 1.

In the first session, after recording the first $\mathrm{H}$-reflex recruitment curve, the inoperative vibrator was applied to the Achilles tendon for $1 \mathrm{~min}$ and in the second session; the inoperative vibrator was applied to the bulk of the soleus muscle. In order to apply the vibrator to the same points in different sessions and different participants, we used osseous landmarks. To determine the bulk of the soleus muscle, resisted plantar flexion was adopted and the vibrator was applied midway between the two heads of the gastrocnemius muscle on the bulk of the soleus. The vibrator was applied to the Achilles tendon $8 \mathrm{~cm}$ above the calcaneal tuberosity. In the first and second experimental sessions, inoperative vibrator was applied to the predetermined points. Immediately after the application of the vibrator and 5 min later, the H-reflex recruitment curve was recorded again.

Matlab software (version 7.2) was used to extract the peakto-peak amplitudes of the H-reflex and $\mathrm{M}$-wave at different intensities. Then, other recruitment curve parameters, including the threshold and the positive slope of the $\mathrm{H}$-reflex recruitment curve, were determined. 
The analysis of the threshold and the ascending slope of the $\mathrm{H}$-reflex recruitment curve was performed by two methods: using a two-point fit (slope calculation by the first two points) and the five-point fit (slope calculation by five points: beginning of the H-reflex, maximum H-reflex, and end point of the H-reflex, plus one point between the first two parameters and another point between the second two parameters) analyses.

\section{Statistical Analysis}

All data were analyzed with Statistical Package for the Social Sciences version 11 (SPSS Inc., IBM Comp, IL, USA) statistical package. Before the paired t-test was performed, variables were tested for normal distribution with the Kolmogorov-Smirnov test. Whenever data showed normal distribution, differences were analyzed by repeated measures analysis of variance (ANOVA) and the paired $t$-test. Statistical significance was assumed if $p$ values were $<0.05$.

\section{Results}

Ten healthy men participated in our study. The data of one subject was excluded from the analysis due to more than $10 \%$ variability in the H-reflex amplitude and one case did not continue after the first two sessions.

To differentiate fast and slow MNs activities, the ascending slope of the $\mathrm{H}$-reflex recruitment curve was analyzed in both two-point and five-point fits. There were no any significant difference between the control and experimental groups at any time (before, immediately after, and 5 min after applying vibration) in the two-point fit analysis $[F(19.1)=0.749$, $p=0.7]$.

As expected, there were no significant differences in the control groups among the three times (before applying sham vibration, immediately after sham vibration, and 5 min after applying sham vibration) in the five-point fit analysis. Means of the five-point fit ascending slope of the $\mathrm{H}$-reflex recruitment curve were significantly different after applying vibration to the tendon of the soleus muscle [ $F(19.1)=93.62, p=0.001]$. Means of the ascending slope increased from $1.23 \pm 0.69 \mathrm{mV} / \mathrm{mA}$ before applying vibration to $1.43 \pm 0.89 \mathrm{mV} / \mathrm{mA}$ immediately after applying vibration and to $1.48 \pm 0.85 \mathrm{mV} / \mathrm{mA} 5 \mathrm{~min}$ after applying
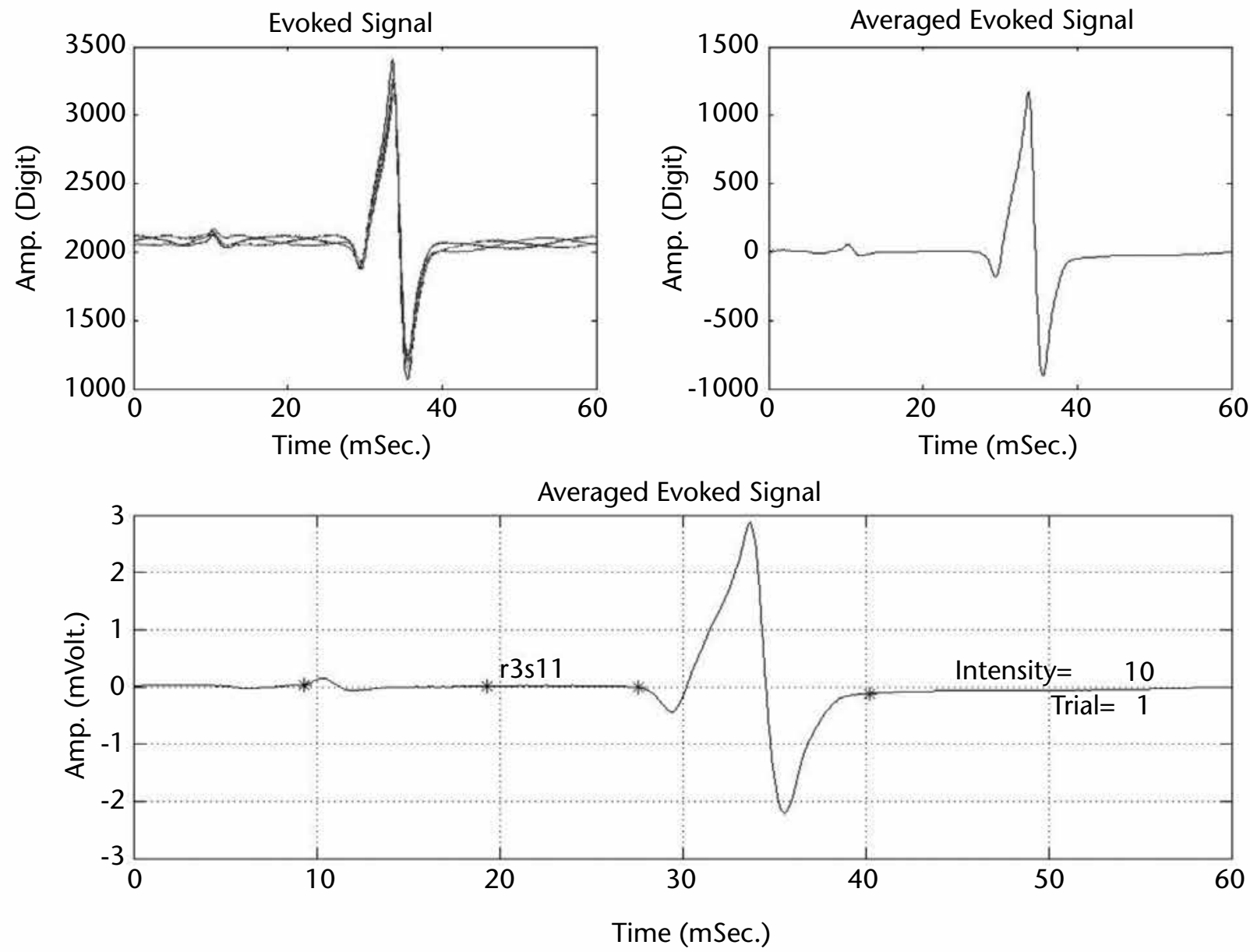

Figure 1. Hmax after the application of vibration

Upper left: five records together, Upper right: average of five records, Below: filtered signal 
vibration. There was an incremental pattern of change in the five-point fit of the ascending slope.

Means of the five-point fit ascending slope of H-reflex recruitment curve were also significantly different after application of vibration to the bulk of soleus muscle $[F(19.1)=4.52$, $\mathrm{p}=0.026]$. Means of the ascending slope increased from $1.29 \pm 0.81 \mathrm{mV} / \mathrm{mA}$ before applying vibration to $1.51 \pm 0.95$ $\mathrm{mV} / \mathrm{mA}$ immediately after applying vibration and to $1.65 \pm 0.95$ $\mathrm{mV} / \mathrm{mA} 5 \mathrm{~min}$ after applying vibration. As can be seen, there was an incremental pattern of change in five-point fit of the ascending slope.

Although there were no significant differences among the three times in the control (sham) groups for the threshold of $\mathrm{H}$ reflex recruitment curve $[F(19.1)=5.52, p=0.28]$, a decremental pattern can be traced for both control groups.

There were statistically significant differences among the three times in tendon experiment group $[F(19.1)=0.58$, $p=0.028]$. Means of the threshold value of the $\mathrm{H}$-reflex decreased from $7.59 \pm 1.62 \mathrm{~mA}$ before applying vibration to $7.38 \pm 1.57 \mathrm{~mA}$ immediately after applying vibration and to $7.26 \pm 1.5 \mathrm{~mA} 5 \mathrm{~min}$ after applying vibration.

In addition, there were statistically significant differences among the three times in the bulk muscle group $[F(19.1)=0.62$, $\mathrm{p}=0.04]$. Means of the H-reflex threshold decreased from $7.59 \pm 1.44 \mathrm{~mA}$ before applying vibration to $7.48 \pm 1.47 \mathrm{~mA}$ immediately after applying vibration and to $7.23 \pm 1.28 \mathrm{~mA} 5 \mathrm{~min}$ after applying vibration.

\section{Discussion}

In the present study, the ascending slope of the H-reflex recruitment curve was analyzed using two-point and five-point fits.

According to the "size" principle, at lower intensities, only slow MNs of the soleus muscle are excited and this follows the weak-strong rule (25). This means that the effect of slow MNs on Renshaw cells is weak, but Renshaw cells exert a strong inhibitory effect on these MNs (26). Vibration excites fast and slow MNs concurrently (27).

At lower intensities, excitement of Renshaw cells, secondary to slow MN activities, increases and these cells inhibit fast MNs in addition to slow ones. Thus, no change is seen in the means of the two-point fit of the ascending slope of the $\mathrm{H}$-reflex recruitment curve. By increasing intensity, more fast MNs are recruited, attenuating the inhibitory effects of Renshaw cells due to recruitment of more fast $\mathrm{MNs}$ (26).

In contrast to the low-intensity state, Renshaw cells cannot inhibit all the fast MNs, so alteration of ascending slope is more apparent in the five-point fit. Therefore, in the evaluation of the ascending slope of the $\mathrm{H}$-reflex recruitment curve, the five-point fit reveals much more than two-point fit. An additional reason is that, similar to other interneurons, Renshaw cells have numerous inputs and outputs, and most of the inputs are excitatory and enhance Renshaw cell activity.

In contrast, applying high-frequency vibration for $1 \mathrm{~min}$, reduces Renshaw cell activity due to a phenomenon called "postactivation depression" (10), and the decreased threshold may be attributable to supraspinal effects as well (28).
Alterations of the threshold of the $\mathrm{H}$-reflex recruitment curve were statistically significant after application of vibration on the bulk and tendon of the soleus muscle. Threshold alterations had a decremental pattern and shifted to the left.

Threshold alterations can be attributed to supraspinal centers. Vibration excites Pacinian corpuscles, and these receptors convey their information through gracile and cuneate fascicles to higher centers. These two fascicles ascend ipsilaterally and terminate in the same nuclei. From there arcuate fibers arise and then terminate in contralateral VPL nuclei.

Gracile and cuneate fascicles also involve la neuronal afferents from muscle spindles and Ib neuronal afferents from GTOs. This system is called the dorsal column medial lemniscus. It is worth noting that la and $\mathrm{lb}$ neuronal afferents from lower extremities terminate on the cerebellum too $(1,29)$.

It is possible that vibration affects the cerebellum in addition to the sensory cortex and these effects inhibit Renshaw cells.

Lyshenko and his colleagues attributed vibration-induced inhibition of Renshaw cells to supraspinal centers (22).

Armstrong et al. (24) attributed the acute effect of WBV on the Hoffmann reflex to factors such as training specificity and muscle fiber type.

\section{Conlusion}

It seems that the application of vibration to the bulk and tendon of the soleus muscle enhanced the positive slope and threshold of the $\mathrm{H}$-reflex recruitment curve.

It can also be concluded that in evaluation of the ascending slope of the $\mathrm{H}$-reflex recruitment curve, the five-point fit is much more reliable than the two-point fit.

Ethics Committee Approval: Ethics committee approval was received for this study from the ethics committee of Tarbiat Modares University (D52/4752).

Informed Consent: Was obtained from patients who participated in this study.

Peer-review: Externally peer-reviewed.

Author contributions: Concept - A.K.Y., G.T.; Design A.K.Y., G.T.; Supervision - G.T.; Data Collection and/or Processing - A.K.Y.; Analysis and/or Interpretation - A.K.Y., G.T., S.M.F; Writing - A.K.Y.; Critical Reviews - G.T., S.M.F.

Conflict of Interest: No conflict of interest was declared by the authors.

Financial Disclosure: This project was supported by a grant from the Postgraduate Studies and Research Program, physical therapy department, faculty of Medical Sciences in Tarbiat Modares University, Tehran, Iran.

\section{References}

1. Umphred D. Neurological rehabilitation. St. Louis: Mosby; 2001.

2. Downie PA. Cash's textbook of Neurology for Physiotherapists. London: Faber and Faber; 1986. 
3. Eklund G, Hagbarth KE. Normal variability of tonic vibration reflexes in man. Exp Neurol 1966; 16:80-92. [CrossRef]

4. Roll JP,Gilhodes JC,Tardy-Gervet MF. Effets perceptifs et moteurs des vibrations musculaires chez l'homme normal: mise en evidence d'une reponse des muscles antagonists.Arch. Ital Biol 1980; 118:51-71.

5. Oh SJ. Clinical Electromyography. Philadelphia: Lippincott Williams \& Wilkins; 2003.

6. Johnson E. Practical Electromyography. Baltimore: Williams \& Wilkins; 1988.

7. Kimura J. Electrodiagnosis in diseases of nerve and muscle: Principles and practice. Philadelphia: F.A. Davis Company; 1989.

8. De Gail P, Lance JW, Neilson PD. Differential effect on tonic and phasic reflex mechanisms produced by vibration of muscles in man. J Neurol Neurosurg Psychiat 1966;29:1-11. [CrossRef]

9. Hagbarth KE, Eklund G. Muscular afferents and motor control. Stockholm, Almqvist \& Wiksell; 1966.

10. Morin C, Pierrot-Deseilligny E. Evidence for presynaptic inhibition of muscle spindle la afferents in man. Neurosci Lett 1984;44:137-42. [CrossRef]

11. Voerman GE, Gregoric M, Hermans HJ. Neurophysiological methods for assessment of spasticity: The Hoffmann reflex, the tendon reflex and the stretch reflex. Disabil Rehabil 2005;27:33-68. [CrossRef]

12. Ali AA, Sabbahi MA. H-reflex under spinal loading and unloading conditions in normal subjects. Clin Neurophysiol 2000;111:66470. [CrossRef]

13. Milner B, Stein B. The contractile properties of human motor units during voluntary isometric contraction. J Physiol (London) 1973;228:285-306. [CrossRef]

14. Hodes R. Effects of age, consciousness and other factors on human electrically induced reflexes (EIRs). EEG Clin Neurophysiol 1967; supp 25:80-91.

15. Tavborikova $\mathrm{H}$, Sax DS. Conditioning of $\mathrm{H}$-reflexes by preceding sub threshold H-reflex stimulus. Brain 1969;92:203-12. [CrossRef]

16. Kameyama $\mathrm{O}$. Methodological considerations contributing to variability of quadriceps H-reflex. Am J Phys Med Rehabil 1989;68:277-82. [CrossRef]
17. Verrier MC. Alterations in H-reflex magnitude by variations in baseline E.M.G. excitability. EEG Clin Neurophysiol 1985;60:492-9. [CrossRef]

18. Landau WM, Clare MH. Fusimotor function. Part 4.Reinforcement of H-reflex in normal subjects. Archieves Neurology 1964;10:11722. [CrossRef]

19. Bell kR, Lehmann JF. Effect of cooling on $\mathrm{H}$ - and T-reflexes in normal subjects. Arch Phys Med Rehabil 1987;68:490-3.

20. Campbell N, Murphy C, Caulfield B, Ward T. Characterization of the Hoffman reflex using mechanography. Electromyography \& Kinesiology 2008;11:299-305.

21. Hilgevoord AA. Normalization of soleus H-reflex recruitment curves in controls and a population of spastic patients. Electroencephalogr Clin Neurophysiol 1994;93:202-8. [CrossRef]

22. Lyshenko VP, Murzin OB, Melrikova OZ. Recurrent inhibition of flexor muscles motoneurons of the man forearm research under vibration and static muscle straininfluence. J Physiol 2000;46:44-9.

23. Beekhuizen K, Field-Fote E. Acute Effects of Whole-Body Vibration on Soleus H-Reflex. Medicine and Science in Sports and Exercise 2004;36:339-60. [CrossRef]

24. Armstrong WJ, Holly NN. The acute effect of whole - body vibration on the Hoffmann reflex. J Strength and Conditioning Research 2008; 22:471-6. [CrossRef]

25. Wakeling JM, Uehil K, Rozitis Al. Muscle fiber recruitment can respond to the mechanics of the muscle contraction. J R Soc Interface 2006;3:533-44. [CrossRef]

26. Hultborn $\mathrm{H}$, Lipski J, Mackel R, Wigstrom H. Distribution of recurrent inhibition with motor nucleus. I Contribution from slow to fast motor units to the excitation of Renshaw cells. Acta Physiol Scand 1988; 134:347-61. [CrossRef]

27. Ribot-Ciscar E, Roll JP, Gilhodes J C. Human motor unit activity during post-vibratory and imitative voluntary muscle contractions. Brain Research 1996;716:84-90. [CrossRef]

28. Chen XY, Chen L, Wolpaw JR. Time course of H-reflex conditioning in the rat. Neuroscience Letters 2001;302:85-8. [CrossRef]

29. Williams PL, Warwick R, Dyson M. Gray's anatomy. Edinburgh: Churchill Livingstone; 1989. 